\title{
A point mutation of mitochondrial genes in diabetes and deafness with focal segmental glomerular sclerosis
}

Received 17 September 2020

Accepted 14 February 2021

Jin Zhang ${ }^{1}$, Yan Wang ${ }^{1}$, Yonggui $\mathrm{Wu}^{1,2, *}$

Department of Nephrology, The First Affiliated Hospital of Anhui Medical University, Hefei, Anhui Province, China

${ }^{2}$ The Center for Scientifc Research of Anhui Medical University, Hefei, Anhui Province, China

Abstract

Deafness, diabetes and proteinuria are typically understood to be an uncommon combination. Here, we reported a 26-year-old woman with a history of persistent deafness, diabetes mellitus, and proteinuria. The diagnosis mainly depends on clinical symptoms, but the cause of the disease should be examined. The histological finding in renal biopsy showed secondary focal segmental glomerular sclerosis (FSGS), but not classic diabetic nephropathy. Further pathogeny was found. Subsequently, a 3243A>G mutation in the mitochondrial DNA was found. Thus, the diagnosis of maternally inherited deafness and diabetes (MIDD) was considered. Ineffective and unnecessary immunosuppression can be avoided through timely diagnosis. Long-term treatment of CoQ10 can be useful in MIDD patients.

Keywords

maternally inherited deafness and diabetes $• \mathrm{FSGS} \cdot$ mitochondrial DNA mutation $\bullet$ CoQ10

\section{Introduction}

Maternally inherited diabetes and deafness (MIDD) affect about $1 \%$ of patients with diabetes [1]. The common clinical manifestations include diabetes mellitus, deafness, emaciation, and maternally inherited traits. Other abnormalities commonly include cardiac disease, renal disease, ophthalmic disease, gastrointestinal disease, myopathies, and short stature [2]. Unfortunately, it can often go misdiagnosed solely as Type 1 or Type 2 diabetes. Timely diagnosis can facilitate the formulation of a correct therapeutic regimen. Here, we report a case of secondary focal segmental glomerular sclerosis (FSGS) caused by MIDD. Informed consent was obtained from the patient for the publication of this case report.

\section{Case Report}

A 26-year-old woman was admitted to our department because of diabetes mellitus and proteinuria, which she had been having for 6 months and 2 months, respectively. Whether it is classic diabetic nephropathy or something special is a question which remains to be explored. Further and close examination of the question indicated that a plausible diagnosis could be drawn from the fact that the patient had bilateral hearing loss and sensorineural hearing loss since she was 16-year-old; however, this condition had been left untreated. In addition, her mother was diagnosed with diabetes mellitus and had passed away a few years prior. Her history was negative for renal disease, hepatitis, tuberculosis, hypertension, and drug allergies.

The physical examination yielded a height of $158 \mathrm{~cm}$ and weight of $41.5 \mathrm{~kg}$ corresponding to a body mass index (BMI) of $16.6 \mathrm{~kg} / \mathrm{m}^{2}$. Her resting blood pressure and pulse were $110 / 72 \mathrm{mmHg}$ and $82 / \mathrm{min}$, respectively. There was no rash, lymphadenopathy, or edema. Other examination on the skin, chest, abdomen, extremities, and nervous system were unremarkable.

The patient's lab results revealed serum creatinine $70.8 \mu \mathrm{mol} / \mathrm{L}$, estimated glomerular filtration rate (GFR) $107 \mathrm{~mL} /\left(\mathrm{min} / 1.73 \mathrm{~m}^{2}\right)$, and albumin $40 \mathrm{~g} / \mathrm{L}$. She presented with proteinuria of $1.4 \mathrm{~g}$ a day, but there were no casts or crystals in the urine. The urine culture of bacteria produced a negative reaction. Other laboratory data including hepatitis, the immunization, and immunofixation electrophoresis were normal. Ultrasonography of kidney revealed normal dimension with left $101 \times 35 \mathrm{~mm}$ and right $104 \times 36 \mathrm{~mm}$. The PE and lab examinations were summarized in Table 1.

Renal biopsy was performed to identify the cause of her proteinuria. Histology of the renal core needle biopsy identified 28 glomeruli, 15 of which were ischemic sclerosis with multifocal atrophy of renal tubules, whereas 2 glomeruli showed segmental sclerosis with glass droplet lesion. In the remaining glomeruli, mild mesangial widening due to an increase in the mesangial matrix (Figure 1A,B) could be observed. Further, focal tubular atrophy and interstitial fibrosis were observed. Interstitial inflammatory infiltrates were generally minimal. Immunofluorescence revealed deposition of $\lg G$ and no deposition of $\lg A$, IgM, C3, C1q, Kappa, and Lambda on the glomerular basement membrane or in the mesangial area (Figure 2). Electron microscopy revealed a mild increase in the mesangial matrix and no obvious changes on the glomerular basement membrane. Serendipity, 


\begin{tabular}{llll}
\hline PE & & \multicolumn{2}{l}{ Lab examinations } \\
\hline BMI & $16.6 \mathrm{~kg} / \mathrm{m}^{2}$ & Scr & $70.8 \mu \mathrm{mol} / \mathrm{L}$ \\
Blood & $110 / 72 \mathrm{mmHg}$ & GFR & $107 \mathrm{~mL} /$ \\
pressure & & & $\left(\mathrm{min} / 1.73 \mathrm{~m}^{2}\right)$ \\
Pulse & 82/min & Albumin & $40 \mathrm{~g} / \mathrm{L}$ \\
& & Proteinuria & $1.4 \mathrm{~g} /$ day \\
& & Left kidney & $101 \times 35 \mathrm{~mm}$ \\
& & Right kidney & $104 \times 36 \mathrm{~mm}$ \\
\hline
\end{tabular}

BMI, body mass index; GFR, glomerular filtration rate.

Table 1. The PE and lab examinations of the patient

an segmental fusion of foot process, and morphologically abnormal mitochondria were observed (Figure 3). A renal pathological diagnosis of secondary FSGS was reached.

Based on diabetes, deafness, secondary FSGS, and the patient's family history of diabetes, maternally inherited deafness and diabetes (MIDD) was under consideration. The molecular genetic analysis of the patient was arranged. To elucidate, blood was obtained and mitochondrial DNA was extracted and whole $m t D N A$ sequencing analysis of patient was performed. A point mutation at position 3243 (A3243G) of the mtDNA was indicated. The rate of mutation is $43.64 \%$, which confirmed the diagnosis of MIDD. In addition to insulin and valsartan, $150 \mathrm{mg}$ of co-enzyme Q10 daily were provided to the patient. The urine protein was attenuated to $0.5 \mathrm{~g}$ a day; this reduction in the amount of protein was evident 3 months later. Proteinuria $<0.5 \mathrm{~g}$ a day lasted nearly for 1 year. A longer-term follow-up was arranged.

\section{Discussion}

In our case, the patient was diagnosed with diabetes and deafness first. The imbalance between proteinuria and the brief history of diabetes mellitus puzzled us. Renal biopsy showed no classic features of diabetic nephropathy but subsequently indicated FSGS. Hildebrandt and coworkers have suggested that every family with a child who has FSGS deserves to be offered an opportunity to undergo a genetic diagnosis (RE) [3].

Based on diabetes, deafness, secondary FSGS, and her family history of diabetes, MIDD was under consideration. Whole mtDNA sequencing analysis of patient showed a point mutation at position 3243 (A3243G) of the mitochondrial DNA. The mitochondrial gene tRNA Leu (UUR) A3243G mutation occupied $85 \%$ of all pathogenic mutations. It will be more complete if the history and the mtDNA analyses of the patient's family members could be showed.

The first MIDD syndrome was reported in 1992 by Reardon $\mathrm{W}$ et al [4]. MIDD patients have a high prevalence of end stage renal disease. The most prevalent finding of renal biopsies is FSGS [5]. Proteinuria, cystic kidney disease, and tubulointerstitial nephropathy were also the other manifestations [6]. Even some cases of renal disease associated with deafness in MIDD have been misdiagnosed as Alport syndrome [7].

MIDD is frequently misdiagnosed by clinicians. Genetic abnormalities in mitochondrial DNA have caused defects in oxidative production of energy [8]. Renal disease may precede the diagnosis of either diabetes or deafness or even be the sole manifestation of the m.3243A $>G$ mutation. The other manifestations of $m .3243 A>G$ in MIDD contained the following, namely central neurological and psychiatric features, ophthalmic disease, myopathy, cardiac disorders, renal disease. endocrine disease, gastrointestinal disease, and miscellaneous features [9].

Although rare, timely and correctly diagnosis of MIDD is important, since it may affect the therapeutic strategy. CoQ10 acted as an electron carrier of the respiratory chain in mitochondria and improved the mutation-associated dysfunction of the respiratory chain in mitochondria [10]. Long-term CoQ10 treatment prevents progressive insulin secretory defect, exercise intolerance, and
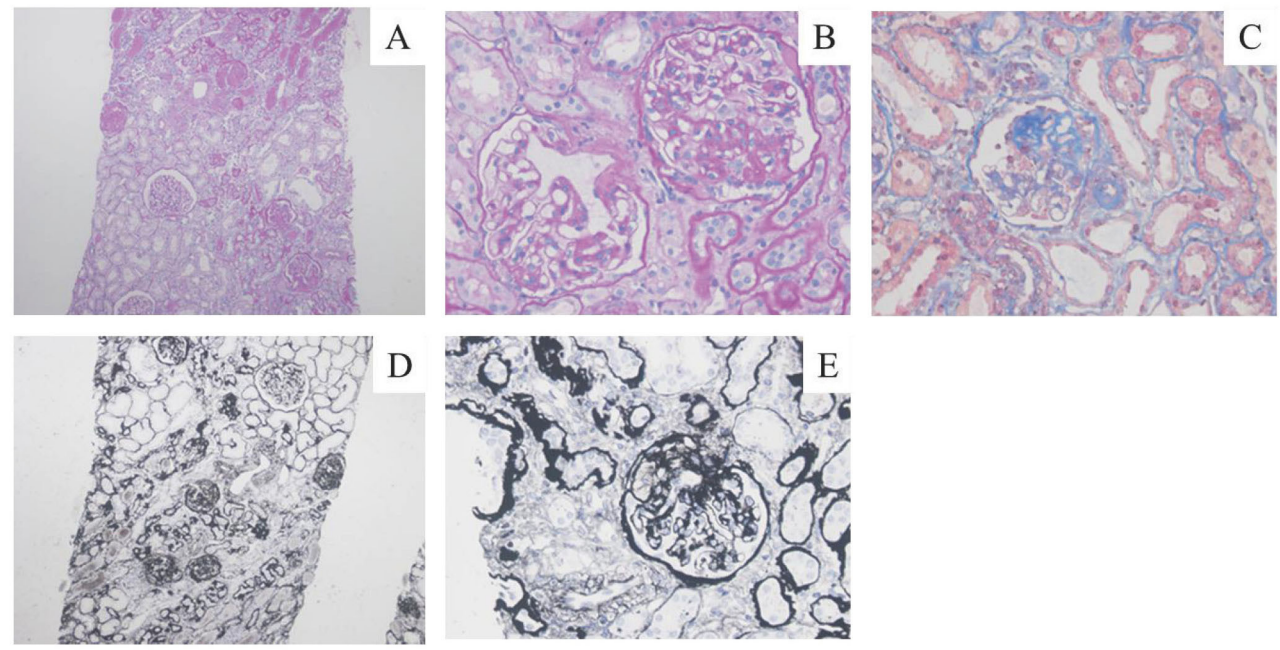

Figure 1. Light microscopic examination. Ischemic sclerosis with multifocal atrophy of renal tubules and segmental sclerosis with glass droplet lesion and interstitial fibrosis could be observed. (A: PAS*100; B: PAS*400; C: Masson*400; D: PASM*100; E: PASM*400). 

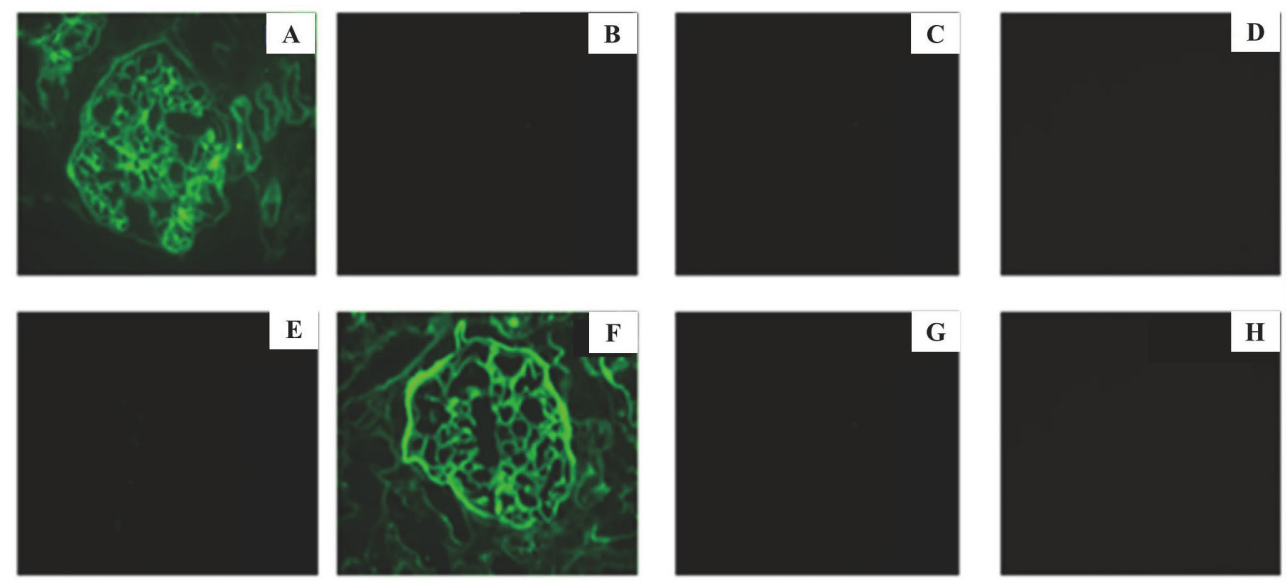

Figure 2. Immunofluorescence on the glomeruli. (A: IgG; B: IgA; C: IgM; D: C3; E: C1q; F: Alb (contral); G: Kappa; H: Lambda).
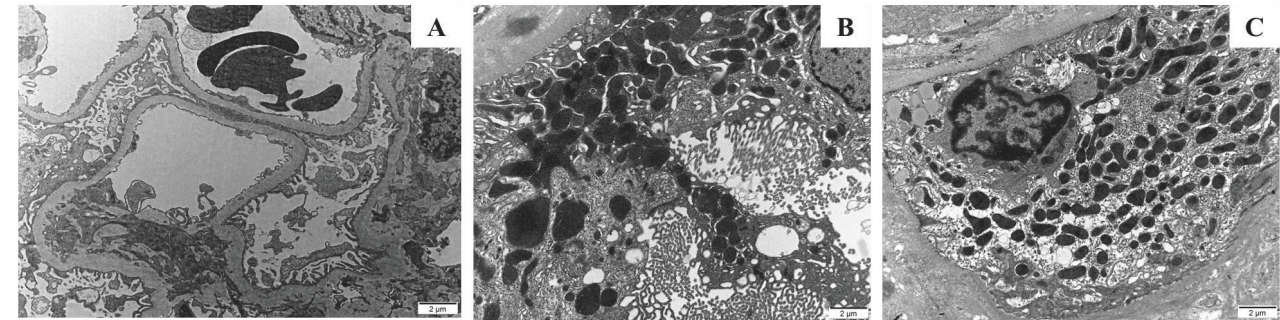

Figure 3. The electron microscopic analysis of the renal tissues (×4000). Segmental fusion foot processes and morphologically abnormal mitochondria were observed. A mild increase in the mesangial matrix could be observed, but no obvious changes on the glomerular basement membrane were noticeable.

hearing loss in MIDD patients. In our case, CoQ10 treatment has been attempted and longer-term follow-up should be arranged.

To conclude, in patients with clinical manifestations of renal disease, particular proteinuria, if deafness and diabetes

\section{Conflict of Interest}

The authors state that they have no conflicts of interest.

\section{Informed Consent}

Patient informed consent has been obtained.

\section{REFERENCES}

[1] Maassen JA, 'T Hart LM, Van Essen E, Heine RJ, Nijpels G, Tafrechi RSJ, et al. Mitochondrial diabetes: Molecular mechanisms and clinical presentation. Diabetes 2004; 53:103-9.

[2] Robinson KN, Terrazas S, Giordano-Mooga S, Xavier NA. The role of heteroplasmy in the diagnosis and management of maternally inherited diabetes and deafness. Endocr Pract 2020; 26(2):241-6.

[3] Sadowski CE, Lovric S, Ashraf S, Pabst WL, Gee HY, Kohl S, et al. A single-gene cause in $29.5 \%$ of cases of steroid-resistant nephrotic syndrome. J Am Soc Nephrol 2015; 26:1279-89. mellitus are also presenting in the course of treatment, MIDD should be differentiated. Ineffective and unnecessary immunosuppression can be avoided through timely diagnosis. Long-term CoQ10 treatment may be useful in MIDD patients.
[4] Reardon W, Ross RJ, Sweeney MG, Luxon LM, Pembrey ME, Harding $\mathrm{AE}$, et al. Diabetes mellitus associated with a pathogenic point mutation in mitochondrial DNA. Lancet 1992; 340:1376-9.

[5] Cao XY, Wei RB, Wang YD, Zhang XG, Tang L, Chen XM. Focal segmental glomerulosclerosis associated with maternally inherited diabetes and deafness: Clinical pathological analysis. Indian J Pathol Microbiol 2013; 56:272-5.

[6] Godinho I, Gameiro J, Jorge S, Abreu F, Neves M, Lopes JA, et al. Diabetes, deafness and renal disease. Clin Kidney J 2017; 10:487-9. 
[7] Guery B, Choukroun G, Noel L, Clavel P, Rötig A, Lebon S, et al. The spectrum of systemic involvement in adults presenting with renal lesion and mitochondrial tRNA (Leu) gene mutation. J Am Soc Nephrol 2003; 14:2099-108.

[8] Cui H, Kong Y, Zhang H. Oxidative stress, mitochondrial dysfunction, and aging. J Signal Transduct 2012; 2012:646354.

[9] Geng X, Zhang Y, Yan J, Chu C, Gao F, Jiang Z, et al. Mitochondrial
DNA mutation m.3243A $>\mathrm{G}$ is associated with altered mitochondrial function in peripheral blood mononuclear cells, with heteroplasmy levels and with clinical phenotypes. Diabet Med 2019; 36:776-83.

[10] Bergamin CS, Rolim LC, Dib SA, Moisés RS. Unusual occurrence of intestinal pseudo obstruction in a patient with maternally inherited diabetes and deafness (MIDD) and favorable outcome with coenzyme Q10. Arq Bras Endocrinol Metabol 2008; 52:1345-9. 